\title{
REDIRECIONAMENTO DE ATAQUES DE NEGAÇÃO DE SERVIÇO PÓS-ANTI-HONEYPOT EM AMBIENTE SDN
}

\author{
Thiago José Lucas ${ }^{1}$, Rodolfo Mura Ferrari ${ }^{1}$, Vinícius Andrade Ribeiro ${ }^{1}$, Miguel Jose das Neves ${ }^{1}$ \\ e Kelton Augusto Pontara da Costa ${ }^{2}$ \\ ${ }^{I}$ Faculdade de Tecnologia - Fatec Ourinhos, Ourinhos - SP, Brasil \\ ${ }^{2}$ UNESP, Universidade Paulista Julio de Mesquita, Bauru - SP, Brasil
}

\begin{abstract}
RESUMO
A quantidade de crimes digitais vem crescendo a cada dia mais, fazendo surgir assim novos métodos de segurança em redes de computadores, métodos estes que têm por objetivo monitorar, controlar e prevenir esses crimes de ocorrerem. Como os criminosos digitais se aperfeiçoam a cada dia, a segurança em redes também deve melhorar suas técnicas para que se possa prevenir a ocorrência destes ataques. O objetivo desse Trabalho é propor um ambiente virtualmente prático, com fundamentação teórica pertinente, que permita que um tráfego malicioso seja afastado da rede local em produção para uma rede virtual. A pesquisa foi realizada por intermédio de artigos, livros, revistas digitais, monografias e sites da área de segurança da informação e redes de computadores, com base nessas pesquisas o trabalho realizou uma simulação de um ataque de força bruta em um servidor Asterisk e a real prevenção desse ataque. Como resultado a pesquisa mostrou que a utilização dessa técnica é eficaz na prevenção desse ataque a redes de computadores, podendo manter assim a segurança da rede em produção preservada. Baseado nas análises feitas na fase prática desse trabalho pode-se comprovar a real importância da implementação de novos métodos de segurança em servidores Asterisk que executam em ambientes SDN.
\end{abstract}

\section{PALAVRAS-CHAVE}

SDN, Honeypot, IDS, Asterisk, Força Bruta

\section{INTRODUÇÃO}

Com os crescentes avanços tecnológicos pelos quais a área de segurança da informação vem passando nos últimos anos, métodos de ataques a redes de computadores também evoluíram. Uma técnica muito utilizada no passado para proteção da rede foi o uso de Honeypots, que consiste na criação de uma área virtual que contém elementos similares aos de uma rede real, a qual seria atacada por um cracker, podendo assim isolar a rede operante da atacada.

Em paralelo ao surgimento dessas novas técnicas surgiu o conceito de Redes Definidas por Software, ideologia técnica que permite centralizar o controle de uma rede de computadores utilizando da divisão de responsabilidades físicas e lógicas, onde o encaminhamento de pacotes é feito por dispositivos de rede sem autonomia de controle independente, e as regras de fluxo em um controlador remoto, por meio do uso do protocolo OpenFlow.

A tendência, de acordo com McGillicuddy (2014) é que em 2016, 87\% das empresas dos Estados Unidos da América possuam, em produção, Redes Definidas por Software. Trata-se de um número expressivo, que deve atrair a atenção e os esforços dos profissionais para a segurança destas redes. Levando-se em consideração as afirmações de Krawetz (2004) de que o uso de Honeypots não é mais eficaz devido à utilização de técnicas Anti-Honeypot, e de que existem Sistemas de Detecção de Intrusão que são capazes de perceber anomalias que podem definir um ataque, o objetivo deste projeto de pesquisa é responder ao problema, que questiona a possibilidade de se somar estes conceitos, a fim de que se possa detectar um ataque cujo processamento prévio (Anti-Honeypot) ocorreu na rede real e desviar seu fluxo via comandos OpenFlow para uma rede de Honeypot. Um ataque proveniente da Internet chega até a rede, o qual pode ser detectado pelo IDS de host (HIDS). O HIDS invocaria a Controladora SDN de que uma anomalia foi detectada, onde a própria Controladora seria responsável por se comunicar com os elementos de rede (como 
switch ou roteador, por exemplo) para que seja realizado o desvio do tráfego do atacante para uma área virtual. Deixando assim a rede operante totalmente isolada da rede virtual, onde nela seria possível a coleta de estatísticas provenientes do ataque.

\section{SOFTWARE-DEFINED NETWORKS}

McKeown et al (2008) deixam claro quando explicam que uma das motivações para o surgimento das Redes Definidas por Software foi a ideia de uma rede a qual seu administrador pudesse separar, em equipamentos distintos, as regras de encaminhamento de pacotes, do encaminhamento propriamente dito.

Segundo Kreutz et al (2015) e Nunes et al (2014), as Redes Definidas por Software (SDN) chegaram com um novo conceito que promete flexibilizar e simplificar, de uma forma drástica, o gerenciamento de redes, permitindo uma evolução e inovação ainda mais rápidas. Os autores complementam afirmando que o modelo SDN é muito similar às redes tradicionais, onde são compostas por uma série de equipamentos, switches, roteadores, que realizam encaminhamento de pacotes. O diferencial das Redes Definidas por Software é a presença de uma controladora, a qual é baseada em planos de controle. Podendo assim deixar restrito aos equipamentos de encaminhamento realizar apenas a tarefa de encaminhar pacotes pela rede, deixando-os livres de incorporação de softwares, e até mesmo controles de tomada de decisão. Onde seu desempenho fica apenas no nível de hardware e a lógica da rede apenas para a controladora.

McKeown et al (2008) completa Kreutz et al (2015) e Nunes et al (2014) onde diz que devido à existência de equipamentos de interface proprietária, fica difícil a compatibilidade e interoperabilidade entre equipamentos de diversos fabricantes. $\mathrm{O}$ autor ainda afirma que em um cenário onde as redes são definidas por software, o protocolo OpenFlow é muito utilizado para realizar a comunicação entre os dispositivos de encaminhamento de pacotes, switches, roteadores, e a controladora. Isso faz com que um único protocolo seja o meio de comunicação entre eles, resolvendo o problema da utilização de equipamentos na rede de diversos fabricantes, onde em certos casos pode ocorrer algum tipo de incompatibilidade de comunicação.

Costa (2013) adverte que as Redes Definidas por Software são muito mais que definições de processamento lógico, como quais são definidas pelo protocolo OpenFlow. Esse protocolo faz parte da aplicação lógica do conceito de SDN, juntamente com o desenvolvimento de aplicações apropriadas para as regras de negócio da empresa, manipulação da rede logicamente ao invés de fisicamente, tudo isso abrange seu conceito, não apenas o processamento lógico.

\subsection{Protocolo openflow}

McKeown et al (2008) dizem que o protocolo OpenFlow foi originalmente proposto pela universidade de Stanford com o propósito de atender as novas arquiteturas e protocolos provenientes de equipamentos comerciais. Baseado principalmente em conceitos de fluxo, onde se tem a combinação de determinados campos do cabeçalho do pacote que virá a ser processado pelo equipamento, onde uma determinada ação será tomada.

Para Costa (2013), uma das características básicas do padrão OpenFlow é a separação clara entre os planos de dados e de controle nos elementos de comutação. O plano de dados é responsável pelo encaminhamento dos pacotes associando entradas na tabela de encaminhamento de cada comutador.

Amorim (2014) diz que atualmente os switches trabalham com endereços de memória para realizar suas tarefas, desde encaminhar pacotes, até políticas de segurança. Entretanto, esse modelo "engessado" não se aplica a equipamentos que trabalham com o protocolo OpenFlow, onde se é possível realizar todas essas ações e ainda programar conforme a necessidade de negócio. O mesmo autor explica de uma forma detalhada as ideias propostas por McKeon et al. (2008) onde diz que um equipamento que não trabalha com o protocolo OpenFlow tem tanto o encaminhamento de pacotes, que é chamado de data path, como as tomadas de decisões, control path, são feitas diretamente no próprio equipamento. Por outro lado, switches que trabalham com o protocolo OpenFlow não realizam o control path, apenas o data path. A controladora é a responsável por se comunicar com o switch, por meio de mensagens, a fim de escolher quais ações deverá tomar. 


\section{SIMULADOR MININET}

O conceito da ferramenta Mininet é descrito por Costa (2013),

Mininet é uma ferramenta para a simulação de Redes Definidas por Software que permite a rápida prototipação de uma grande infraestrutura virtual de rede com a utilização de apenas um computador. O Mininet também possibilita a criação de protótipos de redes virtuais escaláveis baseados em software como OpenFlow utilizando primitivas de virtualização do Sistema Operacional. Com essas primitivas, ele permite criar, interagir e customizar protótipos de Redes Definidas por Software de forma rápida.

Costa (2013) explica de uma forma simples e completa o que é a ferramenta, expõe vantagens de se utilizá-la para testes de Redes Definidas por Software.

Costa (2013) afirma ainda que a Mininet é capaz de simular todo o conceito de Redes Definidas por Software, utilizando o protocolo OpenFlow, onde consegue criar desde estações com interface de rede, as quais ainda estão disponíveis com terminais semelhantes ao de distribuições Linux, a controladora responsável pelas regras de tráfego, os equipamentos que encaminham pacotes, tudo isso simulado dentro de um único computador.

Csoma (2014) corrobora com as palavras de Costa (2013) onde diz que o Mininet é uma ferramenta de código aberto, sem custo, onde é possível criar ambientes independentes de testes em redes SDN complexas, e ainda permitir que seus usuários criem programas específicos para que a simulação acabe se tornando o mais próximo de sua real estrutura. Podendo se perceber as inúmeras vantagens da ferramenta.

Costa (2013) afirma ser possível abrir terminais de comando semelhantes ao de distribuições Linux dentro da ferramenta, Keti e Shavan (2015) explicam alguns dos comandos possíveis de serem executados nesses terminais de comando.

Conforme as referências bibliográficas citadas nessa seção e confirmando por Law e Kelton (1991), um ambiente o qual se pode implementar mudanças, realizar testes de curto e longo prazo, comportamento de uma nova tecnologia desenvolvida, tudo sem afetar a produtividade da empresa é de suma importância no quesito financeiro para a organização.

\section{SISTEMAS E RECURSOS}

\subsection{Sistemas de Detecção e Prevenção de Intrusão}

Segundo Scarfone e Mell (2007), detecção de intrusão é o processo de monitorar os eventos que ocorrem em um sistema ou uma rede de computadores e analisá-los para sinais de possíveis incidentes, em que são violações ou ameaças iminentes de violação das políticas de segurança, ou boas práticas de segurança da informação. Ainda de acordo com os autores, um Sistema de Detecção de Intrusão (IDS) é um software que automatiza o processo de detecção de intrusão. Conforme os autores Sabahi e Movaghar (2008), intrusões em sistemas de computadores geralmente são causadas por atacantes acessando os sistemas pela Internet, ou por usuários internos que tentam exceder os privilégios atribuído a eles.

De acordo com Planquart (2001), sistemas de detecção e prevenção de intrusão podem ser classificados em três categorias:

(i) Host-Based IDPS: avalia informação encontrada em um único ou múltiplos sistemas, incluindo conteúdo de sistemas operacionais, arquivos de aplicativos e sistemas.

(ii) Network-Based IDPS: avalia informação capturada de comunicação em rede, analisando o fluxo de pacotes em tráfego pela rede. Pacotes são capturados através de um conjunto de sensores.

(iii) Vulnerability-Assessment IDPS: detecta vulnerabilidades em redes internas e firewalls.

Em nosso cenário, utilizamos um IPS que, além de detectar possíveis intrusões, também previne que os intrusos consigam comprometer o computador ou a rede de computadores. Além disso, optamos por utilizar um IPS baseado em host porque o objetivo é monitorar um serviço em específico de apenas um único host. 


\subsection{Anti-honeypot}

Um Honeypot é um recurso computacional onde a intenção é ser explorado, atacado ou comprometido. O valor de um Honeypot é determinado pela relevância da informação obtida por ele. Esse recurso não possui valor de produção, por isso qualquer tentativa de acesso é suspeita. Ao ser explorado por um atacante, é possível verificar as teclas digitadas por ele em uma sessão mesmo que o tráfego da rede seja criptografado (Provos et al, 2014).

O Honeypot é usado como isca na forma de sistema vulnerável para emboscar atacantes e mantê-los longe da informação crítica do sistema principal. Nesta técnica, um atacante, inicialmente detectado por um Sistema de Detecção de Intrusão, será reencaminhado para uma rede Honeypot física ou virtual para uma investigação mais detalhada, e tudo isso sem sua percepção (Deepa; Kavitha, 2012). Já Anti-Honeypots são técnicas ou ferramentas usadas para identificar Honeypots e evitar ser emboscado.

Zou e Cunningham (2006) sugeriram um método de revelar Honeypots e removê-los de Botnets. Neste método, o Botmaster comanda Bots nas máquinas comprometidas a executarem ações ilícitas, como Spamming ou realizar requisições contínuas de algum serviço para que pareçam um ataque DDoS . O alvo dessas ações são máquinas dominadas pelo Botmaster que servem de sensores para detectar se o ataque foi bem sucedido. Esta técnica presume que Honeypots não respondem a esses comandos do Botmaster. Portanto, se Honeypots são projetadas para rejeitar estes comandos, então podem ser facilmente detectados pelo Botmaster.

Se usuários maliciosos podem detectar Honeypots, então eles podem evitá-los. No mínimo, essa habilidade poderia diminuir o valor da informação armazenada porque Honeypots evitados não detectariam novos ataques. Mais importante ainda, se pessoas podem detectar um Honeypot, elas podem atacá-lo. Administradores geralmente colocam estes sistemas em LANs adjacentemente com redes críticas. Ao comprometer o sistema, o atacante poderia usá-lo para realizar ataques internos. Ao invés de comprometer o Honeypot, o usuário malicioso poderia também optar por inundar o sistema com informações falsas. Isto efetivamente esconderia qualquer informação de valor sob um excesso de informação inútil. Ao evitar o Honeypot, o atacante previne o sistema de coletar suas informações, além disso, o permite a coletar informações do próprio sistema. Assim como o Honeypot fornece informações valiosas sobre um atacante a seu administrador, um atacante poderia também aprender muito sobre o administrador (Krawetz, 2004).

\section{VULNERABILIDADES DO VoIP}

Apesar das vantagens, a tecnologia VoIP sofre problemas como complexidade de arquitetura, problemas de interoperabilidade, dificuldades de QoS, e problemas de segurança (PARK, 2008). Entre essas desvantagens, os problemas de segurança estão se tornando cada vez mais sérios porque dispositivos comuns de segurança, protocolos, e arquiteturas podem não se adequar corretamente para proteger sistemas VoIP de ataques de segurança recentes (Lazzez, 2013).

As vulnerabilidades no sistema VoIP envolvem não apenas a aplicação em si, mas também nos sistemas operacionais subjacentes, aplicações, e protocolos que o VoIP depende. A complexidade do VoIP faz com que um grande número de vulnerabilidades afetem os três pilares da segurança da informação: confidencialidade, integridade, e disponibilidade (Mcgann; Sicker, 2005).

Mensagens SIP podem conter informações que um usuário ou um servidor desejaria manter em privado. Por exemplo, os cabeçalhos podem revelar informações sobre as partes comunicadoras ou outras informações confidenciais. O corpo da mensagem SIP pode conter informações do usuário (endereços e número de telefone) que não devem ser expostos. As arquiteturas abertas e distribuídas do VoIP, em conjunto com a variedade de subsistemas que a ferramenta fornece, torna o estabelecimento de um ambiente seguro em uma tarefa extremamente difícil (Geneiatakis et al, 2005).

A ameaça mais severa em um ambiente VoIP é provavelmente o acesso fácil ao canal de comunicação. Por exemplo, existem várias ferramentas para análise de tráfego de VoIP, como o WireShark (www.wireshark.org), que torna a espionagem da comunicação uma tarefa fácil para qualquer potencial intruso. O uso de mensagens maliciosas SIP, é também uma possibilidade e pode causar acesso não autorizado ou negação de serviço. Considere, por exemplo, um usuário malicioso que insere um código 
malicioso de SQL em uma mensagem SIP de registro, induzindo alterações não autorizadas no serviço de banco de dados (Geneiatakis et al, 2005).

Um ataque de força bruta, algumas vezes, pode ocasionar uma negação de serviço, pois inúmeras solicitações de logins incorretos podem tornar um servidor instável devido a quantidade de informações que o mesmo terá que processar. As estatísticas do CERT.br mostram que a quantidade de ataques de negação de serviço cresceram muito nos últimos anos: em 2011 foram notificados apenas 272 incidentes relacionados a negação de serviço, enquanto que em 2013 foram notificados 1.030 incidentes do mesmo tipo de ataque, em 2014 esse mesmo número foi 217 vezes maior que no ano anterior, totalizando 223.935 incidentes, e em 2015 foram 25.360 incidentes. Em razão dessas estatísticas, optamos por utilizar esse tipo de ataque neste projeto.

\section{CRIAÇÃO DO CENÁRIO PROPOSTO PARA A REALIZAÇÃO DOS TESTES}

Foi criado um ambiente virtual por meio do software virtualizador Oracle VM VirtualBox, onde se propõe o cenário descrito na Figura 1.

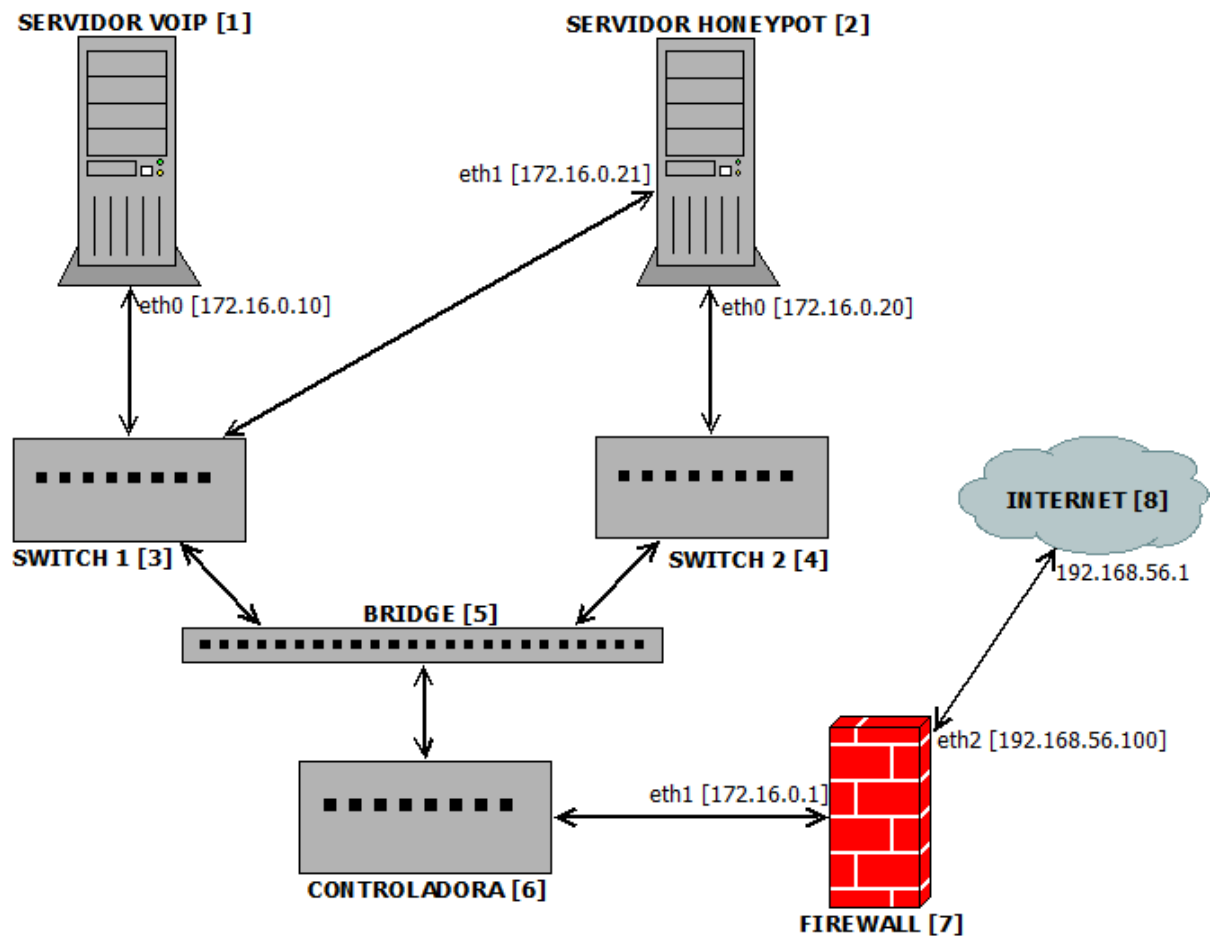

Figura 1. Proposta de cenário para testes

Fonte: Elaborado pelos autores

Todos os dispositivos na rede, com exceção do Firewall, foram executados sobre o simulador de rede MiniNet na versão 2.2.1 que estava a executar sobre uma distribuição Linux Ubuntu 14.04.

O Servidor VoIP [1] teve a função de executar o software Asterisk na versão 11.7.0 para permitir ligações telefônicas através da Internet. Este servidor também executava o Fail2ban na versão 0.8.11, que é um Sistema de Prevenção de Intrusão baseado em Host, responsável por detectar e prevenir ataques de força bruta advindos da Internet. O Fail2ban atua analisando os registros de certa aplicação a fim de banir IPs que tentarem um número excessivo de logins sem êxitos em um curto período de tempo, que muito provavelmente se trata de um ataque de força bruta. 
O Servidor Honeypot [2] também possuía o Asterisk na versão 11.7.0 em execução, porém com o propósito único de servir como uma armadilha. Todo atacante detectado pelo IDS do Servidor VoIP [1] teria o tráfego alterado para o Servidor Honeypot [2] sem que o invasor percebesse, fazendo com que o sistema autêntico ficasse seguro.

O Switch 1 [3] e o Switch 2 [4] possuem suas funções de comutar pacotes entre seus diversos nós como um Switch comum, porém estes possuem o protocolo OpenFlow em operação permitindo assim que fossem gerenciados pela Controladora [6].

O equipamento Bridge [5] possuía a função de servir como uma ponte, assim proporcionando a capacidade de interligar redes distintas.

A Controladora [6] era responsável por gerenciar os Switches 1 e 2 através do protocolo OpenFlow, podendo assim alterar suas tabelas de fluxo com base em critérios definidas pelo administrador da rede.

O Firewall [7] era encarregado de filtrar o tráfego e aceitar apenas os pacotes que respeitassem as políticas definidas pelo administrador.

A Internet [8] é o conjunto de todas as redes que de alguma maneira podem trocar informações com a rede interna. É por esse motivo que se deve tomar muita atenção com o tráfego entre a rede interna e externa. Usuários mal-intencionados podem estar tentando, de alguma forma, comprometer os dados de alguma rede ou host. Ataques de negação de serviço, roubo e sequestro de dados, vírus e malwares são alguns dos muitos ataques que podem proceder da Internet.

\section{REALIZAÇÃO DOS TESTES E RESULTADOS}

Para fazer com que o Fail2ban monitorasse o serviço de VoIP, foi necessário realizar algumas alterações em dois arquivos de configuração da ferramenta. A primeira alteração foi a criação de um arquivo para determinar qual ação o Fail2Ban deverá tomar ao identificar um ataque. A ação definida foi utilizar a ferramenta ovs-ofctl, que auxilia no gerenciamento de Switches OpenFlow, para modificar o fluxo do tráfego atacante com destino ao servidor VoIP para um servidor Honeypot. A ferramenta funciona ao enviar para a Controladora comandos para o gerenciamento de Switches OpenFlow, como configuração de tabelas de fluxos. A Figura 2 mostra a configuração dessa ação.

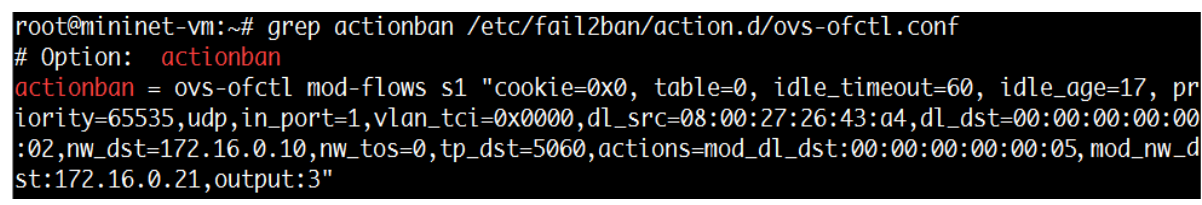

Figura 2. Configuração da ação com seus parâmetros Fonte: Elaborado pelos autores

Ainda no servidor VoIP, foi necessário definir uma Jail para permitir que o Fail2ban monitore o arquivo de log do Asterisk em busca de falhas em logins. A Figura 3 mostra a configuração da Jail. Foram definidas algumas variáveis, e cada uma possui sua função.

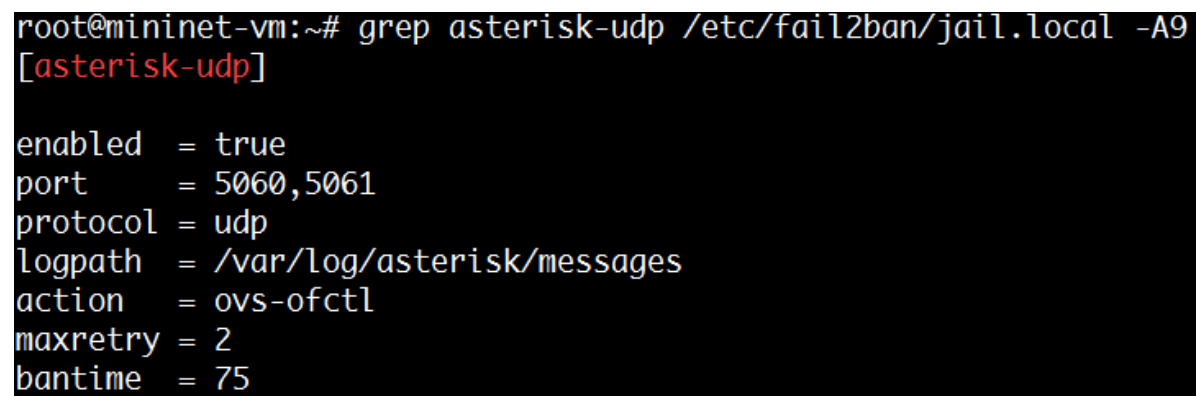

Figura 3. Configuração da Jail

Fonte: Elaborado pelos autores 
Após essas configurações, foi essencial reiniciar o Fail2ban, como exibe a Figura 4.

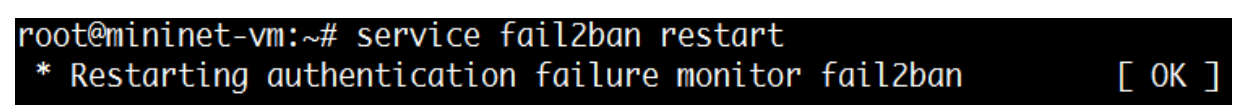

Figura 4. Reinício do Fail2ban

Fonte: Elaborado pelos autores

Para simular um ataque de força bruta, foi utilizado o software gratuito Zoiper, uma ferramenta que permite conectar a um servidor SIP. Para fins de teste apenas, foi especificado na Jail do Fail2ban que seria tolerado no máximo apenas duas tentativas de logins sem sucessos. Dessa forma, ao tentar conectar pela terceira vez no servidor VoIP, o atacante seria banido pelo sistema e teria seu tráfego alterado para o servidor Honeypot. O atacante foi simulado através da máquina hospedeira com comunicação com o Firewall por meio da placa de rede exclusiva de hospedeiro (host-only). O domínio foi definido como o Firewall, e o mesmo encaminharia o tráfego para o servidor VoIP através de regras de IPTables, simulando assim um ataque advindo da Internet

Ao realizar o ataque, foi possível conferir os registros do Fail2ban e observar o momento do "ban", como mostra a Figura 5.

root@mininet-vm: \# tail $-\mathrm{F} / \mathrm{var} / \mathrm{log} / \mathrm{fail}$ ban.log
2016-11-03 12:23:21,841 fail2ban.actions: INF0 Set banTime $=75$
2016-11-03 12:23:37,502 fail2ban.actions: WARNING [asterisk-udp] Ban 192.168.56.1

Figura 5. Registro do ataque

Fonte: Elaborado pelos autores

\subsection{Verificação do Resultado dos Testes}

Ao utilizar a ferramenta ovs-ofctl novamente, foi possível verificar a regra inserida na tabela de fluxos do Switch 1. Perceba que não havia regras antes do ataque, a regra foi inserida apenas após o ataque, como mostra a Figura 6.

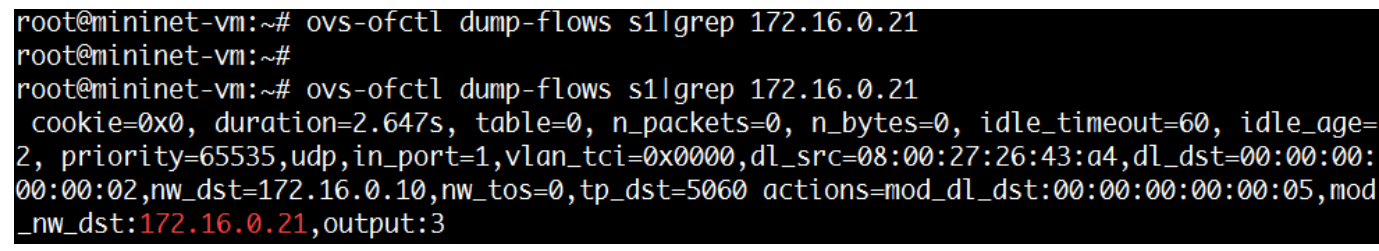

Figura 6. Regra inserida na tabela de fluxo do Switch 1 após o ataque Fonte: Elaborado pelos autores

Por fim, foi possível utilizar o comando tcpdump no servidor Honeypot para monitorar o tráfego encaminhado pela Controladora.

\section{CONCLUSÃO}

Por meio das pesquisas e levantamento dos dados, pode-se inferir a importância de métodos de segurança mais robustos, pois é por meio deles que o administrador da rede consegue manter os pilares da segurança da informação, confidencialidade, integridade e disponibilidade das informações.

A pesquisa revelou que depois da detecção de um ataque de força bruta o tráfego foi realmente desviado. Os comandos necessários para esse desvio foram configurados na Controladora, a qual é responsável pelas regras de tráfego, e sem o atacante perceber foi redirecionado para um servidor Honeypot.

Revelando-se também que a não utilização de novas técnicas por parte dos administradores de rede vêm a acarretar na quebra dos pilares da segurança da informação. 
Como sugestão para trabalhos futuros, no sentido de aperfeiçoamento desta ideia, fica a proposta de que se implemente o cenário proposto com base em NIDS e, em outra vertente, o estudo para redirecionamento de segmentos TCP, que contam com sincronismo e ordenação característicos.

\section{REFERÊNCIAS}

Amorim, R. $O$ uso do protocolo Open Flow em redes definidas pro software. 2014. Disponível em: <http://repositorio.roca.utfpr.edu.br/jspui/bitstream/1/1831/1/CT_GESER_II_2012_09.pdf>. Acesso em Junho, 2016.

CERT.BR. Estatística do CERT.br - Incidentes. 2015. Disponível em: <http://www.cert.br/stats/incidentes/>. Acesso em maio, 2016.

Costa, Lucas Rodrigues. OpenFlow e o paradigma de redes definidas por software. 2013. Disponível em: <http://monografias.cic.unb.br/dspace/bitstream/123456789/391/1/Monografia_Vesao_Leitura_em_PC.pdf >. Acesso em abril de 2016.

Csoma, Attila, et al. ESCAPE: Extensible service chain prototyping environment using mininet, click, netconf and pox. 2014. Disponível em: <http://www.researchgate.net/publication/265731807_ESCAPE _extensible_service_chain_prototyping_environment_using_mininet_click_NETCONF_and_POX>. Acessado em abril de 2016.

Deepa, A. J.; Kavitha, V. A comprehensive survey on approaches to intrusion detection system. Procedia Engineering, v. 38, p. 2063-2069, 2012.

Geneiatakis, Dimitris et al. SIP Security Mechanisms: A state-of-the-art review. In: Proceedings of the Fifth International Network Conference (INC 2005). 2005. p. 147-155.

Keti, F.; Shavan A. Emulation of Software Defined Networks Using Mininet in Different Simulation Environments. Proceedings of the 2015 6th International Conference on Intelligent Systems, Modelling and Simulation. IEEE Computer Society, 2015.

Krawetz, Neal. Anti-honeypot technology. Security \& Privacy, IEEE, v. 2, n. 1, p. 76-79, 2004.

Kreutz, D. ; Ramos, F.M.V. ; Esteves Verissimo, P. ; ESTEVE Rothenberg, C. ; AZODOLMOLKY, S. ; UHLIG, S. 2015. Software-Defined Networking: A Comprehensive Survey In Proc of the IEEE, v.103, no.1, pp.14-76.

Law, A. M. \& Kelton, W. D. Simulation modeling \& analysis. Singapura, McGraw Hill, 1991.

Lazzez, Amor. VoIP Technology: Security Issues Analysis. arXiv preprint arXiv:1312.2225, 2013.

Mcgann, Sicker; Sicker, Douglas C. An analysis of security threats and tools in SIP-based VoIP systems. In: Second VoIP security workshop. 2005.

Mcgillicuddy, Shamus. SDN implementation to rise by 2016, but hurdles remain. 2014. Disponível em: <http://searchsdn.techtarget.com/news/2240226484/SDN-implementation-to-rise-by-2016-but-hurdles-remain>. Acesso em: Outubro, 2016

Mckeown, N. et al. OpenFlow: Enabling Innovation in Campus Networks. ACM SIGCOMM Computer Communication Review, v.38, no.2, pp.69-74, 2008.

Nunes, B.A.A. ; Mendonca, M. ; Xuan-Nam Nguyen ; Obraczka, K.; Turletti, T. 2014. A Survey of Software-Defined Networking: Past, Present, and Future of Programmable Networks. IEEE Communications Surveys \& Tutorials, v.16, pp.1617-1634.

Park, Patrick. Voice over IP security. Pearson Education, 2008

Planquart, Jean-Philippe. Application of neural networks to intrusion detection. 2001.

Provos, Niels et al. A Virtual Honeypot Framework. In: USENIX Security Symposium. 2004. p. 1-14.

Sabahi, Farzad; Movaghar, Ali. Intrusion detection: A survey. In: Systems and Networks Communications, 2008. ICSNC'08. 3rd International Conference on. IEEE, 2008. p. 23-26.

Scarfone, Karen; MELL, Peter. Guide to intrusion detection and prevention systems (idps). NIST special publication, v. 800, n. 2007, p. 94, 2007.

Zou, Cliff C.; CUNNINGHAM, Ryan. Honeypot-aware advanced botnet construction and maintenance. In: Dependable Systems and Networks, 2006. DSN 2006. International Conference on. IEEE, 2006. p. 199-208. 